\title{
Bases in Min-Plus Algebra
}

\author{
Syahida Amalia Rosyada ${ }^{1, *}$ Siswanto $^{2}$ Vika Yugi Kurniawan ${ }^{3}$
}

\author{
${ }^{1,2,3}$ Faculty of Mathematics and Natural Science, Sebelas Maret University, Indonesia \\ *Corresponding author. Email: 1syahidaamalia1@gmail.com
}

\begin{abstract}
In classical linear algebra, a basis is a vector set that generates all elements in the vector space and that vector set is a linear independence set. However, the definitions of the linear dependence and independence in min-plus algebra are little more complex given that the min-plus algebra is the linear algebra over the commutative idempotent semiring. The definition of the linear dependence (independence) is used in this paper is GondranMinoux linear dependence (independence). A finite set is Gondran-Minoux linearly dependent if the set can be divided into two sets that form a linear space with an intersection which is not a zero vector. We will define the concept of the bases in min-plus algebra. In this paper also defined the concept of a weak bases and will be shown that the linear dependence (independence) is not needed to form a weak basis. In the last part of the research result's are proven that every basis in a semimodules in min-plus algebra is a weak basis.
\end{abstract}

Keywords: Basis, Linearly independent, Weak basis.

\section{INTRODUCTION}

A semiring $(S,+, \times)$ is a set $S$ with addition $(+)$ and multiplication $(\times)$ operations, such that $(S,+)$ is a commutative semigroup, $(S, \times)$ is semigroup, and $\times$ distributes over + . The linear algebra over the semiring $\quad \mathbb{R}_{\max }=\mathbb{R} \cup\{-\infty\}$ with two binary operations of maximization $(\bigoplus)$ and addition $(\otimes)$ is called max-plus algebra [1]. In $\mathbb{R}_{\max }$ the neutral (identity) element for $\oplus$ and $\otimes$ are $\varepsilon=-\infty$ and $e=$ 0 . Research on max-plus algebra applications has been carried out by several researchers, see [2][3]. Some researchers also expand the concept in max-plus algebra into interval max-plus algebra, see [4][5]. $\mathbb{R}_{\text {max }}$ is idempotent commutative semiring [6] and can be referred to as idempotent semifield [7]. If $\mathbb{R}_{\max }^{n}$ is defined as $\mathbb{R}_{\text {max }}^{n}=\left\{\left(x_{1}, x_{2}, \ldots, x_{n}\right)^{T} \mid x_{i} \in \mathbb{R}_{\text {max }}, i=\right.$ $1,2, \ldots, n\}, \mathbb{R}_{\max }^{n}$ is a semimodules over the semiring $\mathbb{R}_{\text {max }}$ [6]. The elements $\boldsymbol{x}$ in $\mathbb{R}_{\max }^{n}$ are called maxplus vectors [8].

A subset $F$ of a semimodule $V$ over $\mathbb{R}_{\max }$ spans $V$ if every $x \in V$ is a finite linear combination of all element in $F$. A vector set that generates all elements in the vector space and linearly independent then that vector set is called a basis. The are several concept of linear dependence in max-plus algebra as described in [9][10][11]. In [12], they give examples of vector sets in $\mathbb{R}_{\max }^{n}$ that are weakly linearly independent but Gondran-Minoux linearly dependent and Gondran-
Minoux linearly independent but tropically linearly dependent. In this article, we will use the concept of linear dependence in Gondran-Minoux sense such that a finite set is said to be linearly dependent if the set can be divided into two sets that form a linear space with an intersection which is not a zero vector [9].

In the field of mathematics studies there are another semiring beside max-plus algebra is min-plus algebra. Min-plus algebra is the linear algebra over the semiring $\mathbb{R}_{\text {min }}=\mathbb{R} \cup\{+\infty\}$ that equipped with two binary operations minimization $\left(\oplus^{\prime}\right)$ and addition $(\otimes)$ with the neutral (identity) element for $\bigoplus^{\prime}$ and $\otimes$ are $\varepsilon=+\infty$ and $e=0$ [1]. Max-plus algebra is isomorphic to min-plus algebra [13]. Because there are similarities of structure between max-plus and minplus algebra, we can transform the concepts in maxplus algebra to min-plus algebra. In this article we will define the concept of bases in min-plus algebra.

First, we will discuss the concept of semimodules $\mathbb{R}_{\text {min }}^{n}$ over semiring $\mathbb{R}_{\text {min }}$. Next, we will define the concept of linear dependence in Gondran-Minoux sense in min-plus algebra. Using the concept of linear dependence in Gondran-Minoux sense we will define the concept of bases in min-plus algebra. 


\section{BASIC NOTATIONS AND DEFINITION}

We define the min-plus algebra $\mathbb{R}_{\min }$ by $\mathbb{R}_{\min }=$ $\mathbb{R} \cup\{+\infty\}$ with the binary operations $\oplus^{\prime}$ and $\otimes$, $\forall a, b \in \mathbb{R}_{\min }$

$a \oplus^{\prime} b=\min (a, b)$ and $a \otimes b=a+b$.

There are elements $\varepsilon^{\prime}$ and $e$ in $\mathbb{R}_{\text {min }}$ are denoted $\varepsilon^{\prime}=$ $+\infty$ and $e=0$. For any $a, b, c \in \mathbb{R}_{\text {min }}$ following

i. $\quad\left(a \oplus^{\prime} b\right) \oplus^{\prime} c=a \oplus^{\prime}\left(b \oplus^{\prime} c\right)$

$(a \otimes b) \otimes c=a \otimes(b \otimes c)$

ii. $\quad a \oplus^{\prime} b=b \oplus^{\prime} a$

$a \otimes b=b \otimes a$

iii. $\quad\left(a \oplus^{\prime} b\right) \otimes c=(a \otimes c) \oplus^{\prime}(b \otimes c)$

$a \otimes\left(b \oplus^{\prime} c\right)=(a \otimes b) \oplus^{\prime}(a \otimes c)$

iv. $\quad a \bigoplus^{\prime} \varepsilon^{\prime}=a=\varepsilon^{\prime} \oplus^{\prime} a$

v. $\quad a \otimes e=a=e \otimes a$

vi. $\quad a \otimes(-a)=e=-a \otimes a$

vii. $\quad a \otimes \varepsilon^{\prime}=\varepsilon^{\prime}=\varepsilon^{\prime} \otimes a$

viii. $\quad a \oplus^{\prime} a=a$.

To define the linear dependence and basis in minplus algebra we need the definition of semimodule.

Definition 2.1. A semimodule $M$ over the semiring $(S,+, \times)$ is a commutative monoid $(M,+)$ that equipped with scalar multiplication operation

$$
\begin{aligned}
\bullet: S \times M \rightarrow M & \rightarrow \\
(\lambda, x) & \mapsto \lambda \bullet x
\end{aligned}
$$

and for each $\lambda, \mu \in S, x, y \in M$ following

1. $\lambda \cdot(x+y)=(\lambda \bullet x)+(\lambda \bullet y)$

2. $(\lambda+\mu) \cdot x=(\lambda \cdot x)+(\mu \cdot x)$

3. $(\lambda \cdot \mu) \cdot x=\lambda \cdot(\mu \cdot x)$

4. $\mathbf{1} \cdot x=x$

5. $\lambda \bullet \mathbf{0}=\mathbf{0}$.

Let $\quad \mathbb{R}_{\text {min }}^{n}=\left\{\left(x_{1}, x_{2}, \ldots, x_{n}\right)^{T} \mid x_{i} \in \mathbb{R}_{\text {min }}, i=\right.$ $1,2, \ldots, n\}$. For each $\boldsymbol{x}, \boldsymbol{y} \in \mathbb{R}_{\text {min }}^{n}$ and $\lambda \in \mathbb{R}_{\text {min }}$ defined with operation $\oplus^{\prime}$ and scalar multipication $\bullet$ such that

$\boldsymbol{x} \oplus^{\prime} \boldsymbol{y}=\left(x_{1} \oplus^{\prime} y_{1}, x_{2} \oplus^{\prime} y_{2}, \ldots, x_{n} \oplus^{\prime} y_{n}\right)^{T}$

$\lambda \cdot \boldsymbol{x}=\lambda \otimes \boldsymbol{x}=\left(\lambda \otimes x_{1}, \lambda \otimes x_{2}, \ldots, \lambda \otimes x_{n}\right)^{T}$.

In [14], we notice that $\left(\mathbb{R}_{\min }^{n}, \oplus^{\prime}\right)$ is a commutative monoid with the neutral element $\left(\varepsilon^{\prime}, \varepsilon^{\prime}, \ldots, \varepsilon^{\prime}\right)^{T}$ and $\mathbb{R}_{\text {min }}^{n}$ satisfies the axiom in Definiton 2.1 by operations $\bigoplus^{\prime}$ and $\otimes$, therefore $\mathbb{R}_{\min }^{n}$ is semimodule over the semiring $\mathbb{R}_{\text {min }}$.

Definition 2.2. A subset $V$ in $\mathbb{R}_{\min }^{n}$ is a commutative idempotent semimodule over $\mathbb{R}_{\text {min }}$ if it is closed under $\oplus^{\prime}$ and scalar multiplication such that $\boldsymbol{u} \oplus^{\prime} \boldsymbol{v} \in V$ and $\alpha \otimes \boldsymbol{v} \in V, \forall \boldsymbol{u}, \boldsymbol{v} \in \mathbb{R}_{\min }^{n}$ and $\alpha \in \mathbb{R}_{\text {min }}$.

Definition 2.3. A element $x$ is a finite linear combination of elements in $F \subseteq V$ if $x=\oplus^{\prime} \lambda_{f} \otimes \boldsymbol{f}$, such that $\lambda_{f}=\varepsilon^{\prime}$ finetely for some $\boldsymbol{f} \in F$.

\section{RESULT AND DISCUSSION}

Definition 3.1. A subset $F$ of a semimodule $V$ over $\mathbb{R}_{\text {min }}$ generates $V$ if every element $\boldsymbol{x} \in V$ is a finite linear combination of all elements in $F$.

Definition 3.2. A generating set is called minimal if it can be divided into two disjoint subsets such that for some $\alpha_{i} \in \mathbb{R}_{\text {min }}, i \neq k$

$$
\oplus^{\prime} \alpha_{i} \otimes \boldsymbol{v}_{\boldsymbol{i}} \neq \oplus^{\prime} \alpha_{k} \otimes \boldsymbol{v}_{\boldsymbol{k}} \text {. }
$$

Definition 3.3. A family of vetors $\left\{\boldsymbol{u}_{i}\right\}_{i=1}^{p}$ is a weak basis of a semimodule $V$ if it is a minimal generating set.

We will define the concept of linear dependence (independence) in the Gondran-Minoux sense in minplus algebra based on the analogy in [9].

Definition 3.4. Vectors $\boldsymbol{v}_{\mathbf{1}}, \boldsymbol{v}_{\mathbf{2}}, \ldots, \boldsymbol{v}_{\boldsymbol{p}} \in \mathbb{R}_{\min }^{n}$ are called Gondran-Minoux linearly dependent if there exists disjoint subsets $I$ and $K, I \cup K=\{1,2, \ldots p\}$ such that for $\alpha_{j} \neq \varepsilon^{\prime}(j \in I \cup K)$

$$
\bigoplus_{i \in I}^{\prime} \alpha_{i} \otimes v_{i}=\bigoplus_{k \in K}^{\prime} \alpha_{k} \otimes v_{k} .
$$

If no such $I, K$, and $\alpha_{j}$ exist, $\left\{\boldsymbol{v}_{1}, \boldsymbol{v}_{2}, \ldots, \boldsymbol{v}_{\boldsymbol{p}}\right\}$ is a linearly independent set.

Definition 3.5. Vectors $\boldsymbol{v}_{\mathbf{1}}, \boldsymbol{v}_{2}, \ldots, \boldsymbol{v}_{\boldsymbol{p}} \in \mathbb{R}_{\min }^{n}$ are called Gondran-Minoux linearly independent if for all disjoint subsets $I$ and $K, I \cup K=1,2, \ldots p$ and all $\alpha_{j} \in \mathbb{R}_{\min }$

$$
\bigoplus_{i \in I}^{\prime} \alpha_{i} \otimes v_{i} \neq \bigoplus_{k \in K}^{\prime} \alpha_{k} \otimes v_{k}
$$

unless $\alpha_{j}=\varepsilon^{\prime}, \forall j \in I \cup K$.

Using the linear dependence (independence) definition in min-plus algebra, then the following definition is obtained.

Let $W \subseteq \mathbb{R}_{\min }^{n}$ and a nonempty finite subset $U=$ $\left\{\boldsymbol{u}_{1}, \boldsymbol{u}_{2}, \ldots, \boldsymbol{u}_{\boldsymbol{n}}\right\}$ of $W$. For each $\boldsymbol{w} \in W$ can be written as a finite linear combination of all elements of $U$ (denoted by $\boldsymbol{w} \sim U$ ) as in Definition $\mathbf{2 . 3}$ for $\boldsymbol{u}_{\boldsymbol{i}} \neq$ $\boldsymbol{w}, i=1,2, \ldots, n$. The following theorem will explain the reason for there is such an exception $\left(\boldsymbol{u}_{\boldsymbol{i}} \neq \boldsymbol{w}\right)$.

Theorem 3.1. For any $\boldsymbol{x}, \boldsymbol{y} \in \mathbb{R}_{\text {min }}^{n}$, there is a $\lambda \in$ $\mathbb{R}_{\text {min }}$ such that $\boldsymbol{x} \oplus^{\prime} \lambda \otimes \boldsymbol{y}=\boldsymbol{x}$.

Proof. If $\boldsymbol{x}=\left(x_{1}, x_{2}, \ldots, x_{n}\right)^{T}$ and $\boldsymbol{y}=$ $\left(y_{1}, y_{2}, \ldots, y_{n}\right)^{T}$ then for the $\lambda$ we may take any value greater than or equal to the maximum of $\left(x_{1} \otimes\right.$ $\left.y_{1}^{-1}, x_{2} \otimes y_{2}^{-1}, \ldots, x_{n} \otimes y_{n}^{-1}\right)$.

Definition 3.6. Let $V$ be a semimodule in $\mathbb{R}_{\min }^{n}$. A finite subset $U(U \neq \varnothing)$ of the set $V$ is called a basis of $V$ if and only if $U$ is a generating set of $V$ and $U$ is linearly independent in other words for each $v \in V$ either $\boldsymbol{v} \in U$ or $\boldsymbol{v} \sim U$ but no both.

Definiton 3.7. Suppose that $U \subseteq V$ is a basis of a semimodule $V$. The number of vectors in $U$ is called the dimension of $V$ and denoted by $\operatorname{dim}(V)$. 
In [15] Wagneur stated that every a finitely generated semimodule has a weak bases. For any two weak bases have the same number of generators.

Definition 3.8. The weak basis cardinality is called the weak rank of the semimodule $V$ and denoted by $r_{w}(V)$.

Consider the definitions, the following examples are given.

Example 3.1. Given the set $P$ in $\mathbb{R}_{\text {min }}^{3} . P$ is defined as $P=\{(-1, e, e),(e,-1, e),(e, e,-1)\} . P$ is a linear independent set in Gondran-Minoux sense because not exist $\alpha_{j} \neq \varepsilon^{\prime}$ that satisfies $\bigoplus_{i \in I}^{\prime} \alpha_{i} \otimes \boldsymbol{v}_{\boldsymbol{i}}=$ $\bigoplus_{k \in K}^{\prime} \alpha_{k} \otimes \boldsymbol{v}_{\boldsymbol{k}}$. However, it is seen that $P$ does not generate $\mathbb{R}_{\text {min }}^{3}$. Therefore $P$ is not a basis of $\mathbb{R}_{\text {min }}^{3}$.

Example 3.2. Let $X=\left\{\left(e, \varepsilon^{\prime}\right),\left(\varepsilon^{\prime}, e\right),(e, e)\right\}$ be the set in $\mathbb{R}_{\text {min }}^{2}$. Each element of $\mathbb{R}_{\text {min }}^{2}$ is a finite linear combination of $X$ so that it can be written

$$
\begin{aligned}
\mathbb{R}_{\text {min }}^{2}= & \left\{\left(\begin{array}{l}
a \\
b
\end{array}\right) \mid a, b \in \mathbb{R}_{\text {min }}\right\} \\
= & \left\{a \otimes\left(\begin{array}{c}
e \\
\varepsilon^{\prime}
\end{array}\right) \oplus^{\prime} b \otimes\left(\begin{array}{c}
\varepsilon^{\prime} \\
e
\end{array}\right) \oplus^{\prime} \varepsilon^{\prime} \otimes\left(\begin{array}{l}
e \\
e
\end{array}\right) \mid a, b \in\right. \\
& \left.\mathbb{R}_{\text {min }}\right\} .
\end{aligned}
$$

Therefore it is clear that $X$ generates $\mathbb{R}_{\text {min }}^{2}$. There is a vector in $X$ that can be written $e \otimes(e, e)=e \otimes$ $\left(e, \varepsilon^{\prime}\right) \bigoplus^{\prime} e \otimes\left(\varepsilon^{\prime}, e\right)$ which satisfies Definition 3.4. Since $X$ generates $\mathbb{R}_{\min }^{2}$ but is not linearly independent then $X$ is not a basis of $\mathbb{R}_{\min }^{2}$. On other side we can show that $X$ is a weak basis of $\mathbb{R}_{\text {min }}^{2}$ because for any $\alpha, \beta \in \mathbb{R}_{\min }$

$$
e \otimes\left(e, \varepsilon^{\prime}\right) \neq \alpha \otimes(e, e) \oplus^{\prime} \beta \otimes\left(\varepsilon^{\prime}, e\right)
$$

with $r_{w}\left(\mathbb{R}_{\min }^{2}\right)=3$

Example 3.3. Let us consider the following four matrices

$\begin{array}{ll}\boldsymbol{R}=\left(\begin{array}{cc}-1 & \varepsilon^{\prime} \\ \varepsilon^{\prime} & \varepsilon^{\prime}\end{array}\right) & \boldsymbol{S}=\left(\begin{array}{cc}\varepsilon^{\prime} & 1 \\ \varepsilon^{\prime} & \varepsilon^{\prime}\end{array}\right) \\ \boldsymbol{T}=\left(\begin{array}{cc}\varepsilon^{\prime} & \varepsilon^{\prime} \\ -1 & \varepsilon^{\prime}\end{array}\right) & \boldsymbol{U}=\left(\begin{array}{ll}\varepsilon^{\prime} & \varepsilon^{\prime} \\ \varepsilon^{\prime} & e\end{array}\right) .\end{array}$

Suppose that $M=\{\boldsymbol{R}, \boldsymbol{S}, \boldsymbol{T}, \boldsymbol{U}\}$. It will be shown that $M$ is a basis of $\mathbb{R}_{\text {min }}^{2 \times 2}$.

i. $\quad M$ generates $\mathbb{R}_{\text {min }}^{2 \times 2}$.

$$
\begin{aligned}
\mathbb{R}_{\text {min }}^{2 \times 2}= & \left\{\left(\begin{array}{ll}
a & b \\
c & d
\end{array}\right) \mid a, b, c, d \in \mathbb{R}_{\text {min }}\right\} \\
= & \left\{(a+1) \otimes \boldsymbol{R} \oplus^{\prime}(b-1) \otimes \boldsymbol{S} \oplus^{\prime}(c+\right. \\
& \left.1) \otimes \boldsymbol{T} \oplus^{\prime} d \otimes \boldsymbol{U} \mid a, b, c, d \in \mathbb{R}_{\text {min }}\right\} .
\end{aligned}
$$

ii. $M$ is a linerly independent set because not exist $\alpha_{j} \neq \varepsilon^{\prime} \quad$ that satisfies $\bigoplus_{i \in I}^{\prime} \alpha_{i} \otimes M_{\boldsymbol{i}}=$ $\bigoplus_{k \in K}^{\prime} \alpha_{k} \otimes \boldsymbol{M}_{k}$ for $j \in I \cup K, \boldsymbol{M}_{j} \in M$.

So $M$ is a basis of $\mathbb{R}_{\text {min }}^{2 \times 2}$ with $\operatorname{dim}\left(\mathbb{R}_{\text {min }}^{2 \times 2}\right)=4$.

Theorem 3.2. Let $V$ is a finite semimodule in $\mathbb{R}_{\text {min }}^{2}$ and $U \subseteq V$ is a basis of $V$ then $U$ is a weak basis of $V$. Proof. Since $U$ is a basis of $V$ then $U$ is generating set of $V$ and $U$ is linearly independent. $U$ is linearly independent such that it satisfies a minimal generating set. Therefore $U$ is a weak basis of $V$.
Consider the set in Example 3.3, $M$ is a basis of $\mathbb{R}_{\text {min }}^{2 \times 2}$. Using Theorem 3.2 $\boldsymbol{M}$ can be called a weak basis of $\mathbb{R}_{\text {min }}^{2 \times 2}$. Also consider the set in Example 3.2, $X$ is a linearly dependent (not linearly independent) and is a weak basis of $\mathbb{R}_{\text {min }}^{2}$. Because there is a weak basis that is linearly independent or linearly dependent, then the linear independence is not needed to form a weak basis of a semimodule over semiring $\mathbb{R}_{\text {min }}$.

\section{AUTHORS' CONTRIBUTIONS}

The first author contributed to the realization of the research and writing the article script. The second author contributed to generating research ideas, reviewing article writing, and applying for publication funding. The third author contributed to reviewing article writing.

\section{ACKNOWLEDGMENTS}

The authors might want to thank the Sebelas Maret University for financing this research in the academic year of 2021 .

\section{REFERENCES}

[1] K. P. Tam, Optimizing and Approximating Eigenvectors in Max-Algebra. PHD's Thesis, The University of Birmingham, 2010.

[2] L. Prastiwi, Y. Listiana, The Application of MaxPlus Algebra to Deteremine The Optimal Time of Ikat Kupang Woven, in: International Journal of Computing Science and Applied Mathematics, vol. 3(2), 2017, pp. 77-80. DOI: http://dx.doi.org/10.12962/j24775401.v3i2.2317

[3] Subiono, K. Fahim, D. Adzkiya, Generalized Public Transportation Scheduling using MaxPlus Algebra, in: Kybernetika, vol. 54(2), 2018, pp. 243-267. DOI: https://doi.org/10.14736/kyb2018-2-0243

[4] Siswanto, V. Y. Kuniawan, Pangadi, B. S. Wiyono, Characteristic Polynomial of Matrices over Interval Max-Plus Algebra, in: AIP Conference Proceedings, vol. 2326(020033), 2021, pp. 1-5. DOI: https://doi.org/10.1063/5.0039779

[5] Siswanto, A. Suparwanto, M. A. Rudhito, Ruang Vektor Eigen Suatu Matriks Atas Aljabar MaxPlus Interval, in: Jurnal Matematika \& Sains, vol. 19(1), 2014, pp. 8-15. 
[6] M. A. Rudhito, S. Wahyuni, A. Suparwanto, F. Susilo, System of Fuzzy Number Max-Plus Linear Equations, in: Journal of the Indonesian Mathematical Society, vol. 17(1), 2011, pp. 1728.

DOI: https://doi.org/10.22342/jims.17.1.10.17-28

[7] C. Wang, Y. Xia, Y, Tao, Ordered Structures of Polynomial over Max-Plus Algebra, in: Symmetry, vol. 13(7), 2021, pp. 1137. DOI: https://doi.org/10.3390/sym13071137

[8] K. G. Farlow, Max-Plus Algebra. Master's Thesis, Virginia Polytechnic Institute and State University, 2009.

[9] M. Gondran, M. Minoux, Linear Algebra in Dioids: A Survey of Recent Results, in: NorthHolland Mathematics Studies, vol. 95(C), 1984, pp. $\quad 147-163$. DOI: https://doi.org/10.1016/S0304-0208(08)72960-8

[10] W. Vogel, Minimax-algebra, in: European Journal of Operational Research, vol. 4(2), 1980, pp. 144-146. DOI: https://doi.org/10.1016/0377$\underline{2217(80) 90027-2}$

[11] Z. Izhakian, L. Rowen, The Tropical Rank of a Tropical Matrix, in: Communications in Algebra, vol. 37(11), 2009, pp. 3912-3927. DOI: https://doi.org/10.1080/00927870902828793

[12] M. Akian, S. Gaubert, A. Guterman, Linear Independence over Tropical Semirings and Beyond, in: Contemporary Mathematics, vol. 495(1), 2009, pp. 1-38. DOI: http://dx.doi.org/10.1090/conm/495/09689

[13] A. Rosenmann, F. Lehner, A. Peperko, Polynomial Convolutions in Max-plus Algebra, in: Linear Algebra and Its Applications, vol. 578, 2019, pp. 370-401. DOI: https://doi.org/10.1016/j.1aa.2019.05.020

[14] S. Watanabe, Y. Watanabe, Min-Plus Algebra and Networks, in: RIMS Kokyuroku Bessatsu, vol. 47, 2014, pp. 41-54. DOI: https://doi.org/10.1007/springerreference 21127

[15] E. Wagneur, Moduloïds and pseudomodules 1. Dimension theory, in: Discrete Mathematics, vol.98(1), 1991, pp. 57-73. DOI: https://doi.org/10.1016/0012-365X(91)90412-U 